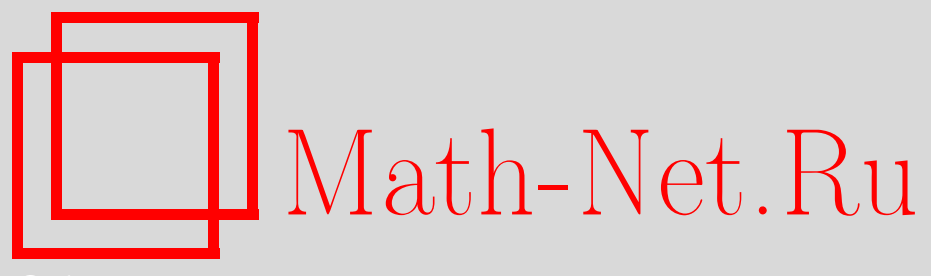

В. А. Клячин, О некоторых свойствах устойчивых и неустойчивых поверхностей предписанной средней кривизны, Изв. РАН. Сер. матем., 2006, том 70, выпуск 4, 77-90

DOI: https://doi.org/10.4213/im587

Использование Общероссийского математического портала Math-Net.Ru подразумевает, что вы прочитали и согласны с пользовательским соглашением http://www . mathnet.ru/rus/agreement

Параметры загрузки:

IP : 34.227 .88 .159

26 апреля 2023 г., 13:32:51

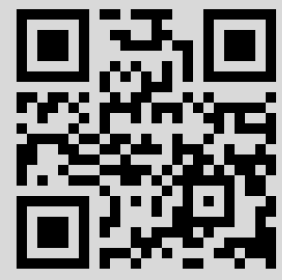




\title{
В. А. Клячин \\ О некоторых свойствах устойчивых и неустойчивых поверхностей предписанной средней кривизны
}

\begin{abstract}
Исследуются свойства устойчивых (и неустойчивых) гиперповерхностей предписанной средней кривизны в евклидовом пространстве. Доказан ряд необходимых и достаточных признаков устойчивости, сформулированных в терминах внешнегеометрического строения поверхности. Получен аналог известной теоремы А.Д. Александрова, обобщающий вариационное свойство сферы. Найдена точная оценка протяженности устойчивой трубчатой поверхности постоянной средней кривизны. Метод исследования базируется на анализе выражений первой и второй вариаций функционалов типа площади рассматриваемых поверхностей.
\end{abstract}

Библиография: 22 наименования.

\section{Введение}

В настоящей работе исследуется проблема устойчивости гиперповерхностей предписанной средней кривизны в евклидовом пространстве. Проблема устойчивости подмногообразий нулевой средней кривизны в евклидовых пространствах и римановых многообразиях изучена весьма глубоко. В последние годы этой проблеме были посвящены статьи Д. Хоффмана, Р. Оссермана, Дж. Барбосы и М. до Кармо, Х. Лоусона, А. В. Погорелова, Дж. Саймонса, А. А. Тужилина, А. Т. Фоменко и др. (см. [1]-[10]).

Минимальные поверхности (поверхности нулевой средней кривизны) суть экстремали функционала площади (первая вариация функционала площади обращается в нуль). Устойчивость минимальной поверхности означает положительную определенность второй вариации функционала площади, и каждая поверхность с нулевой средней кривизной устойчива в достаточно малой окрестности любой своей точки.

В настоящей работе мы определяем специальные функционалы на множестве гладких поверхностей таким образом, что поверхности с предписанной средней кривизной как функции точек пространства являются экстремалями указанных функционалов. Экстремальные поверхности вводимых в работе функционалов моделируют, например, физические равновесные жидкости в гравитационном поле с потенциалом, определяемым функцией средней кривизны $[15, \S 1.4]$. При моделировании тентовых покрытий также возникают поверхности, минимизирующие соответствующий функционал. При этом во всех указанных примерах весьма актуальной является задача определения условий устойчивости или неустойчивости. С физической точки зрения устойчивость той или иной механической системы означает положительную определенность 
второй вариации потенциальной энергии системы. В нашем случае потенциальная энергия выражается определяемыми функционалами. Следует отметить, что некоторые частные случаи экстремальных поверхностей для средней кривизны, зависящей только от одной фиксированной координаты, рассмотрены в работах [9], [11]-[16]. В частности, в работе [9] доказаны признаки неусточивости поверхностей постоянной средней кривизны в терминах интеграла от нормы второй квадратичной формы, однако устойчивость поверхности понимается в несколько ином смысле - относительно вариаций, допускающих изменение объема специальной области, на границе которой расположена сама поверхность. В работах [11]-[16] получены признаки устойчивости в терминах объема такой области для осесимметричных капиллярных поверхностей, расположенных в поле силы тяжести.

Результаты настоящей работы анонсированы в [17].

\section{§ 1. Функционалы и их вторые вариации}

Пусть $\mathbb{R}^{n+1}-(n+1)$-мерное евклидово пространство, в котором введен ортонормированный базис $\left\{e_{i}\right\}_{i=1}^{n+1}$. Через $\langle$,$\rangle мы обозначаем скалярное произ-$ ведение в $\mathbb{R}^{n+1}$. Пусть $x_{1}, x_{2}, \ldots, x_{n+1}$ - соответствующие выбранному базису декартовы координаты.

Согласованную со скалярным произведением связность в $\mathbb{R}^{n+1}$ мы будем обозначать через $\bar{\nabla}$.

Пусть $M-n$-мерное некомпактное связное ориентируемое $C^{2}$-многообразие с кусочно гладким краем $\partial M$ (случай $\partial M=\varnothing$ не исключается). Будем рассматривать поверхности $\mathcal{M}=(M, u)$, заданные $C^{2}$-погружением $u: M \rightarrow \mathbb{R}^{n+1}$.

Связность $\bar{\nabla}$ индуцирует соответствующую связность $\nabla$ на поверхности $\mathcal{M}$. Для произвольных $C^{1}$-гладких векторных полей $X, Y$ на $\mathcal{M}$ и функции $h \in$ $C^{1}(\mathcal{M})$, где $\mathcal{M}=(M, u)$, связность $\nabla$ определяется следующим образом. Рассматриваются произвольные $C^{1}$-продолжения векторных полей $X, Y$ и функции $h$ в некоторую окрестность поверхности $\mathcal{M}$. Тогда

$$
\nabla h=(\bar{\nabla} h)^{T}, \quad \nabla_{X} Y=\left(\bar{\nabla}_{X} Y\right)^{T},
$$

где $(v)^{T}$ - ортогональная проекция вектора $v$ на касательную плоскость $T_{u(m)} \mathcal{M}$ к поверхности $\mathcal{M}$.

Пусть $m \in M$ и в некоторой окрестности точки $u(m)$ определены гладкие векторные поля $X$ и $Y$. Билинейная форма

$$
B(X(m), Y(m))=\left(\bar{\nabla}_{X} Y\right)(u(m))-\left(\bar{\nabla}_{X} Y\right)^{T}(u(m))
$$

называется второй фундаменталъной формой поверхности $\mathcal{M}[19$, т. $2, \S 3]$. Если $\left\{E_{i}\right\}_{i=1}^{n}-$ ортонормированный базис в касательном пространстве к поверхности $\mathcal{M}$ в точке $u(m)$, то вектор

$$
\vec{H}(m)=\frac{1}{n} \operatorname{trace} B=\frac{1}{n} \sum_{i=1}^{n} B\left(E_{i}, E_{i}\right)
$$

называется вектором средней кривизны поверхности $\mathcal{M}$ в точке $u(m)$.

Пусть $N_{u(m)} \mathcal{M}$ - нормальное пространство к поверхности $\mathcal{M}$ в точке $u(m)$. Для произвольного вектора $v \in N_{u(m)} \mathcal{M}$ через $A^{v}$ обозначим гомоморфизм 
Вейнгартена [19, гл. VII, §3], определяемый как линейное преобразование $A^{v}: T_{u(m)} \mathcal{M} \rightarrow T_{u(m)} \mathcal{M}$, двойственное к билинейной форме $B:$

$$
\left\langle A^{v}(X), Y\right\rangle=\langle B(X, Y), v\rangle=-\left\langle\bar{\nabla}_{X} v, Y\right\rangle .
$$

Положим

$$
\left\|A^{v}\right\|^{2}=\sum_{i=1}^{n}\left|A^{v}\left(E_{i}\right)\right|^{2},
$$

где $\left\{E_{i}\right\}_{i=1}^{n}$ - ортонормированный базис в $T_{u(m)} \mathcal{M}$.

Пусть $\Omega \subset \mathbb{R}^{n+1}$ - некоторая область и $\Omega_{1} \subset \Omega$ - ее подобласть такая, что $\partial \Omega_{1} \cap \partial \Omega=\mathcal{M}$. Предположим, что в $\mathbb{R}^{n+1}$ определены неотрицательные $C^{2}$ функции $\alpha(x)$ и $\varphi(x)$.

Будем рассматривать функционалы, выражаемые интегралами

$$
\begin{gathered}
A(\mathcal{M})=\int_{\mathcal{M}} \alpha(x) d x, \quad G(\mathcal{M})=\int_{\Omega_{1}} \varphi(x) d x, \\
W(\mathcal{M})=A(\mathcal{M})+G(\mathcal{M}) .
\end{gathered}
$$

Заметим, что функции $\alpha(x)$ и $\varphi(x)$ с физической точки зрения являются поверхностной и объемной плотностями сил, действующих на элемент жидкости, занимающей объем области $\Omega_{1}$.

Пусть $v$ - сечение нормального расслоения поверхности $\mathcal{M}$. Продолжим поле $v$ до векторного поля $V$ на некоторую окрестность поверхности $\mathcal{M}$, интегральными кривыми которого служат прямые линии. Обозначим через $F_{t}: \mathbb{R}^{n+1} \rightarrow \mathbb{R}^{n+1}$ однопараметрическую группу локальных диффеоморфизмов поля $V$, через $\mathcal{M} t=F_{t}(\mathcal{M})$ - соответствующую ей вариацию поверхности $\mathcal{M}$, а через $\Omega_{1}(t)=F_{t}\left(\Omega_{1}\right)$ - соответствующую вариацию области $\Omega_{1}$. Рассмотрим функции $A(t)=A\left(\mathcal{M}_{t}\right), G(t)=G\left(\mathcal{M}_{t}\right)$. Поверхность назовем экстремальной для функционала $W(\mathcal{M})$, если производная $W^{\prime}(0)$ равна нулю для всякого нормального сечения $v$ с компактным носителем на поверхности $\mathcal{M}$. Здесь $W(t)=A(t)+G(t)$. Кроме этого, если для всех вариаций, порождаемых сечениями $V$ нормального расслоения экстремальной поверхности $\mathcal{M}$, выполнено $W^{\prime \prime}(0)>0$, то поверхность будем называть устойчивой.

Целью настоящей работы является исследование свойств устойчивых экстремальных поверхностей, а также получение ряда признаков устойчивости и неустойчивости. С этой целью мы вычисляем первую и вторую вариации соответствующего функционала $W(\mathcal{M})$. Отметим монографию [15], посвященную исследованию равновесных капиллярных поверхностей, которые в наших терминах моделируются случаем $\varphi(x)=$ const $\cdot x_{3}, n=2$. Устойчивость капиллярных поверхностей вращения в $\mathbb{R}^{3}$ исследована в работе [16].

Теорема 1. Выполнены следующие равенства:

$$
\begin{aligned}
\left.\frac{d W(t)}{d t}\right|_{t=0}= & \int_{\mathcal{M}}\langle\bar{\nabla} \alpha, v\rangle-\alpha n\langle v, \vec{H}\rangle+\varphi\langle v, \xi\rangle d \mathcal{M} \\
\left.\frac{d^{2} W(t)}{d t^{2}}\right|_{t=0}= & \int_{\mathcal{M}}\left\{\left(\alpha\left(|\nabla v|^{2}+\left(n^{2} H^{2}-\|A\|^{2}\right)\right)+(\langle\bar{\nabla} \varphi, \xi\rangle-n H \varphi)\right)\langle v, \xi\rangle^{2}\right. \\
& -2\langle\bar{\nabla} \alpha, v\rangle n H\langle v, \xi\rangle+\operatorname{Hess} \alpha(v, v)\} d \mathcal{M},
\end{aligned}
$$


где $v$ - варьирующее нормальное векторное поле, $\xi$ - внешняя по отношению $\kappa$ области $\Omega$ единичная нормаль к поверхности $\mathcal{M}, H=\langle\vec{H}, \xi\rangle-$ средняя кривизна, $\|A\|$ - длина второй квадратичной формы поверхности $\mathcal{M}, \operatorname{Hess} \alpha(\cdot, \cdot)$ гессиан функции $\alpha$.

ПримеР 1. Пусть объем $\Omega_{1} \subset \Omega \subset \mathbb{R}^{3}$ занимает жидкость, а $\mathcal{M}$ - свободная поверхность этой жидкости (т. е. поверхность жидкости, не соприкасающаяся с твердым телом). Предположим, что жидкость находится в равновесии в гравитационном поле с потенциалом, равным $\varphi(x)$. Если $\alpha=$ const - коэффициент поверхностного натяжения, то полная энергия данной механической системы имеет вид

$$
W_{0}(\mathcal{M})=\alpha|\mathcal{M}|+\int_{\Omega_{1}} \varphi(x) d x,
$$

где $|\mathcal{M}|$ - площадь поверхности. Хорошо известно, что механическая система находится в равновесии, если вариация ее потенциальной энергии равна нулю. Используя теорему 1, последнее условие можно записать в виде

$$
\int_{\mathcal{M}}(\varphi-n \alpha H) h d \mathcal{M}=0
$$

где $h=\langle v, \xi\rangle$. Если мы считаем, что жидкость несжимаема, то последнее соотношение должно быть выполнено не при всех вариациях поверхности $\mathcal{M}$, а только для тех, для которых вариация объема жидкости равна нулю. Как нетрудно видеть (см. теорему 1), последнее имеет место при условии

$$
\int_{\mathcal{M}} h d \mathcal{M}=0
$$

если мы считаем, что жидкость однородна и ее плотность $\rho=1$. Используя основную лемму вариационного исчисления и метод множителей Лагранжа при решении задач условного экстремума, приходим к равенству

$$
\varphi-n \alpha H=\lambda \equiv \text { const, }
$$

или

$$
n \alpha H=\varphi+\lambda .
$$

Таким образом, экстремали функционала $W_{0}$ в рассматриваемом примере имеют среднюю кривизну, определяемую с точностью до постоянной потенциалом $\varphi(x)$. Из теоремы 1 также находим условие устойчивого равновесия:

$$
\int_{\mathcal{M}}\left\{\alpha\left(|\nabla h|^{2}+\left(n^{2} H^{2}-\|A\|^{2}\right)+(\langle\bar{\nabla} \varphi, \xi\rangle-n H \varphi-n H \lambda) h^{2}\right)\right\} d \mathcal{M} \geqslant 0 .
$$

Это неравенство должно быть выполнено для всех $C^{1}$-функций $h(x): \mathcal{M} \rightarrow \mathbb{R}$ таких, что

$$
\left.h(x)\right|_{\partial \mathcal{M}}=0, \quad \int_{\mathcal{M}} h(x) d \mathcal{M}=0 .
$$

Используя равенство нулю первой вариации, это неравенство перепишем в виде

$$
\int_{\mathcal{M}}\left\{\alpha\left(|\nabla h|^{2}+\left(\langle\bar{\nabla} \varphi, \xi\rangle-\|A\|^{2}\right) h^{2}\right)\right\} d \mathcal{M} \geqslant 0 .
$$

Заметим, что условие (4) не зависит от постоянной $\lambda$. 
ОПРЕДЕЛЕНИЕ 1. Поверхность $\mathcal{M}$ назовем экстремалъной поверхностъю для функционала $W_{0}(\mathcal{M})$, если для некоторой постоянной $\lambda$ выполнено равенство

$$
\varphi-n \alpha H=\lambda \equiv \text { const. }
$$

При этом поверхность $\mathcal{M}$ называется устойчивой, если неравенство (4) выполнено для любой $C^{1}$-гладкой функции $h: \mathcal{M} \rightarrow \mathbb{R}$ такой, что выполнены равенства (3).

Далее в статье рассматривается случай функционала $W_{0}(\mathcal{M})$, когда $\alpha \equiv 1$.

Пусть $\mathcal{M}=(M, u)-n$-мерная поверхность в $\mathbb{R}^{n+1}$. Мы введем следующие величины:

$$
\mu_{A}(\mathcal{M})=\inf \frac{\int_{\mathcal{M}}|\nabla h|^{2} d \mathcal{M}}{\int_{M}\|A\|^{2} h^{2} d \mathcal{M}}, \quad \mu(\mathcal{M})=\inf \frac{\int_{\mathcal{M}}|\nabla h|^{2} d \mathcal{M}}{\int_{M} h^{2} d \mathcal{M}}
$$

где точная нижняя грань взята по всем липшицевым функциям $h(m): M \rightarrow \mathbb{R}$ таким, что $\int_{\mathcal{M}} h(m) d \mathcal{M}=0$.

ЗАмечАниЕ 1 . Предположим, что $\varphi(x) \equiv 1$. Тогда экстремали функционала $W_{0}(\mathcal{M})$ суть поверхности постоянной средней кривизны. В этом случае условие устойчивости запишется в виде

$$
\int_{\mathcal{M}}\left\{\left(|\nabla h|^{2}-\|A\|^{2} h^{2}\right)\right\} d \mathcal{M} \geqslant 0 .
$$

Отсюда следует, что поверхность постоянной средней кривизны устойчива тогда и только тогда, когда $\mu_{A}(\mathcal{M}) \geqslant 1$.

Отметим, что для минимальных гиперповерхностей в работе [10] предложен емкостный подход к оценке величины $\mu_{A}(\mathcal{M})$.

Лемма 1. Пусть поверхность $\mathcal{M}$ имеет постоянную среднюю кривизну $H$. Предположим, что найдется положительная $C^{2}$-функиия $u(x)$ : $\mathcal{M} \rightarrow \mathbb{R}$, удовлетворяющая в метрике поверхности $\mathcal{M}$ дифференциальному неравенству

$$
\Delta u(x) \leqslant-\|A\|^{2} u(x) .
$$

Тогда $\mu_{A} \geqslant 1$ и поверхность $\mathcal{M}$ устойчива.

СЛЕДСТВИЕ 1. Поверхность постоянной средней кривизны, заданная графиком функиии, устойчива.

Для более общей ситуации имеет место

ТЕОРема 2. Пусть $\mathcal{M}$ - экстремальная поверхность для функционала $W_{0}(\mathcal{M})$ такая, что $\mu(\mathcal{M})<n \inf _{\mathcal{M}} H^{2}$. Если вдоль поверхности $\mathcal{M}$ выполнено $\langle\bar{\nabla} \varphi, \xi\rangle \leqslant 0$, то $\mathcal{M}$ неустойчива.

Следующая теорема обобщает вариационное свойство сферы и является аналогом известной теоремы А. Д. Александрова [18].

ТЕОРема 3. Устойчивая замкнутая (без края) погруженная экстремальная для функиионала $W_{0}(\mathcal{M})$ поверхность $\mathcal{M}$, вдоль которой $\langle\bar{\nabla} H, \xi\rangle \leqslant 0$, является стандартной евклидовой сферой. 
В следующей теореме утверждается, что слишком длинные трубки постоянной средней кривизны неустойчивы. Физические аспекты данного явления рассмотрены в книге [20].

ТеОремА 4. Пусть $\mathcal{M}$ - погруженная поверхность постоянной средней кривизны $H \neq 0$ в $\mathbb{R}^{n+1}$, граница которой расположена в параллельных гиперплоскостях $\Pi_{1} u \Pi_{2}$, заданных уравнениями $x_{n+1}=a$ u $x_{n+1}=b$ coответственно. Если расстояние межсуу $\Pi_{1}$ и $\Pi_{2}$ удовлетворяет неравенству

$$
b-a>\frac{2 \pi L_{\max }}{\sqrt{n}|H| L_{\min }},
$$

то поверхность $\mathcal{M}$ неустойчива. Если поверхность $\mathcal{M}$ имеет неположительную скалярную кривизну, то $\mathcal{M}$ неустойчива при

$$
b-a>\frac{2 \pi L_{\max }}{n|H| L_{\min }} .
$$

Здесъ $L_{\max }, L_{\min }$ - максимальная и минимальная $(n-1)$-мерные меры сечения поверхности $\mathcal{M}$ плоскостями вида $\left\{x: x_{n+1}=\right.$ const $\}$.

\section{§ 2. Доказательство теоремы 1}

Доказательство теоремы 1 проведем в два этапа. Сначала вычислим первую и вторую производные функции $A(t)$, а затем функции $G(t)$. Обозначим через $\omega(n+1)$-форму объема в $\mathbb{R}^{n+1}$, через $X_{1}, X_{2}, \ldots, X_{n}$ - ортонормированные касательные векторные поля на $\mathcal{M}$, через $\xi$ - векторное поле нормалей для поверхностей $\mathcal{M}_{t}$. Пусть $F_{t} X_{i}=X_{i}^{*}$. Тогда имеем

$$
A(t)=\int_{\mathcal{M}}\left(\alpha \omega\left(X_{1}^{*}, X_{2}^{*}, \ldots, X_{p}^{*}, \xi\right)\right) \circ F_{t}(m) .
$$

Для доказательства нам потребуется

ЛЕмма 2. Если $\bar{\nabla}-$ связность в $\mathbb{R}^{n+1}$, mо $\bar{\nabla} \omega \equiv 0$.

ДоказАтельство. Пусть $\xi_{1}, \xi_{2}, \ldots, \xi_{n+1}$ - ортонормированные векторные поля в $\mathbb{R}^{n+1}$. Тогда для любого $Y \in T N$ имеем

$$
\begin{aligned}
\left(\bar{\nabla}_{Y} \omega\right)\left(\xi_{1}, \xi_{2}, \ldots, \xi_{n+1}\right)= & \bar{\nabla}_{Y}\left(\omega\left(\xi_{1}, \xi_{2}, \ldots, \xi_{n+1}\right)\right)-\omega\left(\bar{\nabla}_{Y} \xi_{1}, \xi_{2}, \ldots, \xi_{n+1}\right) \\
& -\omega\left(\xi_{1}, \bar{\nabla}_{Y} \xi_{2}, \ldots, \xi_{n+1}\right)-\omega\left(\xi_{1}, \xi_{2}, \ldots, \xi_{n}, \bar{\nabla}_{Y} \xi_{n+1}\right) .
\end{aligned}
$$

Ясно, что $\omega\left(\xi_{1}, \xi_{2}, \ldots, \xi_{n+1}\right) \equiv 1$, a $\bar{\nabla}_{Y} \xi_{i}$ ортогонален $\xi_{i}$ и, следовательно, линейно зависит от $\xi_{1}, \xi_{2}, \ldots, \widehat{\xi_{i}}, \ldots, \xi_{n+1}$. Здесь $\widehat{\xi_{i}}$ означает, что символ $\xi_{i}$ пропущен. Из этого заключаем, что $\bar{\nabla}_{Y} \omega\left(\xi_{1}, \xi_{2}, \ldots, \xi_{n+1}\right)=0$. Далее из линейности $\bar{\nabla} \omega$ и того, что $\bar{\nabla}_{Y} \omega-$ тензор, заключаем, что $\bar{\nabla} \omega \equiv 0$.

Продолжим доказательство теоремы. По определению производной Ли [19, т. $1, \S 3]$ :

$$
\begin{aligned}
\frac{d}{d t}\left\{\omega\left(X_{1}^{*}, X_{2}^{*}, \ldots, X_{n}^{*}, \xi\right) \circ F_{t}\right\} & =L_{V}\left\{\omega\left(X_{1}^{*}, X_{2}^{*}, \ldots, X_{n}^{*}, \xi\right) \circ F_{t}\right\} \\
& =\bar{\nabla}_{V} \omega\left(X_{1}^{*}, X_{2}^{*}, \ldots, X_{n}^{*}, \xi\right) \circ F_{t}
\end{aligned}
$$


поэтому

$$
A^{\prime}(t)=\int_{\mathcal{M}} \bar{\nabla}_{V}\left(\alpha\left\{\omega\left(X_{1}^{*}, X_{2}^{*}, \ldots, X_{n}^{*}, \xi\right)\right\}\right) \circ F_{t} .
$$

В силу леммы 2 имеем

$$
\begin{aligned}
A^{\prime}(t)=\int_{\mathcal{M}} & \bar{\nabla}_{V} \alpha\left(\left\{\omega\left(X_{1}^{*}, X_{2}^{*}, \ldots, X_{n}^{*}, \xi\right)\right\}\right) \circ F_{t} \\
& +\sum_{i=1}^{n} \int_{\mathcal{M}} \alpha\left(F_{t}(x)\right) \omega\left(X_{1}^{*}, \ldots, \bar{\nabla}_{V} X_{i}^{*}, \ldots, X_{p}^{*}, \xi\right) \circ F_{t} \\
& +\int_{\mathcal{M}} \omega\left(X_{1}^{*}, X_{2}^{*}, \ldots, X_{p}^{*}, \bar{\nabla}_{V} \xi\right) \circ F_{t} .
\end{aligned}
$$

По построению полей $X_{i}^{*}$ имеем $L_{V} X_{i}^{*}=\bar{\nabla}_{V} X_{i}^{*}-\bar{\nabla}_{X_{i}^{*}} V=0$, а тензор $\bar{\nabla}_{V} \xi$ линейно зависим от $X_{1}^{*}, \ldots, X_{n}^{*}$. Поэтому из равенства (8) получаем

$$
\begin{aligned}
A^{\prime}(t)=\int_{\mathcal{M}} & \bar{\nabla}_{V} \alpha\left(\left\{\omega\left(X_{1}^{*}, X_{2}^{*}, \ldots, X_{n}^{*}, \xi\right)\right\}\right) \circ F_{t} \\
& \quad+\sum_{i=1}^{n} \int_{\mathcal{M}} \alpha\left(F_{t}(x)\right) \omega\left(X_{1}^{*}, \ldots, \bar{\nabla}_{X_{i}^{*}} V, \ldots, X_{n}^{*}, \xi\right) \circ F_{t} .
\end{aligned}
$$

Используя определение вектора $H$, непосредственно из (9) получаем

$$
A^{\prime}(0)=\int_{\mathcal{M}}\langle\bar{\nabla} \alpha, v\rangle-\langle\vec{H}, v\rangle
$$

Дифференцируя (9) еще раз, аналогично получаем

$$
\begin{aligned}
A^{\prime \prime}(t)=\int_{\mathcal{M}} & \bar{\nabla}_{V} \bar{\nabla}_{V} \alpha\left(\left\{\omega\left(X_{1}^{*}, X_{2}^{*}, \ldots, X_{n}^{*}, \xi\right)\right\}\right) \circ F_{t} \\
& +2 \sum_{i=1}^{n} \int_{\mathcal{M}} \bar{\nabla}_{V} \alpha\left(F_{t}(x)\right) \omega\left(X_{1}^{*}, \ldots, \bar{\nabla}_{V} X_{i}^{*}, \ldots, X_{p}^{*}, \xi\right) \circ F_{t} \\
& +\sum_{i=1}^{n} \int_{\mathcal{M}} \omega\left(X_{1}^{*}, \ldots, \bar{\nabla}_{V} \bar{\nabla}_{X_{i}^{*}} V, \ldots, X_{n}^{*}, \xi\right) \circ F_{t} \\
& +\sum_{i \neq j} \omega\left(X_{1}^{*}, \ldots, \bar{\nabla}_{X_{j}^{*}} V, \ldots, \bar{\nabla}_{X_{i}^{*}} V, \ldots, X_{n}^{*}, \xi\right) \circ F_{t} \\
& +\sum_{i=1}^{n} \int_{\mathcal{M}} \omega\left(X_{1}^{*}, \ldots, \bar{\nabla}_{X_{i}^{*}} V, \ldots, X_{n}^{*}, \bar{\nabla}_{V} \xi\right) \circ F_{t} .
\end{aligned}
$$

Далее, учитывая равенства

$$
\begin{aligned}
\omega\left(X_{1}^{*}, \ldots,\right. & \left.\bar{\nabla}_{X_{i}^{*}} V, \ldots, X_{n}^{*}, \bar{\nabla}_{V} \xi\right)\left.\right|_{\mathcal{M}}=\omega\left(X_{1}, \ldots, \bar{\nabla}_{X_{i}} v, \ldots, X_{n}, \bar{\nabla}_{V} \xi\right) \\
& =\omega\left(X_{1}, \ldots, \nabla_{X_{i}} v, \ldots, X_{n}, \bar{\nabla}_{V} \xi\right)-\omega\left(X_{1}, \ldots, A^{v}\left(X_{i}\right), \ldots, X_{n}, \bar{\nabla}_{V} \xi\right)
\end{aligned}
$$




$$
\begin{aligned}
= & \omega\left(X_{1}, \ldots, \nabla_{X_{i}} v, \ldots, X_{n},\left\langle\bar{\nabla}_{V} \xi, X_{i}\right\rangle X_{i}\right) \\
& -\omega\left(X_{1}, \ldots, A^{v}\left(X_{i}\right), \ldots, X_{n},\left\langle\bar{\nabla}_{V} \xi, X_{i}\right\rangle X_{i}\right) \\
= & \omega\left(X_{1}, \ldots,\left\langle\nabla_{X_{i}} v, \xi\right\rangle \xi, \ldots, X_{n},\left\langle\bar{\nabla}_{V} \xi, X_{i}\right\rangle X_{i}\right) \\
= & \omega\left(X_{1}, \ldots, \xi, \ldots, X_{n}, X_{i}\right)\left\langle\nabla_{X_{i}} V, \xi\right\rangle\left\langle\bar{\nabla}_{V} \xi, X_{i}\right\rangle \\
= & \left\langle\nabla_{X_{i}} V, \xi\right\rangle^{2} \omega\left(X_{1}, \ldots, X_{i}, \ldots, X_{n}, \xi\right), \\
\omega\left(X_{1}^{*}, \ldots,\right. & \left.\bar{\nabla}_{X_{i}^{*}} V, \ldots, \bar{\nabla}_{X_{j}^{*}} V, \ldots, X_{n}^{*}, \xi\right)\left.\right|_{\mathcal{M}} \\
= & \omega\left(X_{1}, \ldots, A^{v}\left(X_{i}\right), \ldots, A^{v}\left(X_{j}\right), \ldots, X_{n}, \xi\right) \\
= & \left(\left\langle A^{v}\left(X_{i}\right), X_{i}\right\rangle\left\langle A^{v}\left(X_{j}\right), X_{j}\right\rangle-\left(\left\langle A^{v}\left(X_{i}\right), X_{j}\right\rangle\right)^{2}\right) \omega\left(X_{1}, \ldots, X_{n}, \xi\right), \\
\omega\left(X_{1}^{*}, \ldots,\right. & \left.\bar{\nabla}_{V} \bar{\nabla}_{X_{i}^{*}} V, \ldots, X_{n}^{*}, \xi\right) \\
= & \left.\omega\left(X_{1}^{*}, \ldots, \bar{\nabla}_{V} \bar{\nabla}_{X_{i}^{*}} V-\bar{\nabla}_{X_{i}^{*}} \bar{\nabla}_{V} V-\bar{\nabla}_{\left[V, X_{i}^{*}\right]} V, \ldots, X_{n}^{*}, \xi\right)\right|_{\mathcal{M}}=0,
\end{aligned}
$$

получаем

$$
\begin{aligned}
A^{\prime \prime}(t)=\int_{\mathcal{M}} & \bar{\nabla}_{V} \bar{\nabla}_{V} \alpha\left(\left\{\omega\left(X_{1}^{*}, X_{2}^{*}, \ldots, X_{n}^{*}, v\right)\right\}\right) \circ F_{t} \\
& +2 \sum_{i=1}^{n} \int_{\mathcal{M}} \bar{\nabla}_{V} \alpha\left(F_{t}(x)\right) \omega\left(X_{1}^{*}, \ldots, \bar{\nabla}_{V} X_{i}^{*}, \ldots, X_{p}^{*}, v\right) \circ F_{t} \\
& +\sum_{i \neq j} \alpha\left(F_{t}(x)\right)\left(\left\langle A^{v}\left(X_{i}\right), X_{i}\right\rangle\left\langle A^{v}\left(X_{j}\right), X_{j}\right\rangle-\left(\left\langle A^{v}\left(X_{i}\right), X_{j}\right\rangle\right)^{2}\right) \\
& +\alpha\left(F_{t}(x)\right) \sum_{i=1}^{n}\left(\left\langle\nabla_{X_{i}} v, \xi\right\rangle\right)^{2} .
\end{aligned}
$$

Используя определение средней кривизны, находим

$$
\sum_{i \neq j}\left(\left\langle A^{v}\left(X_{i}\right), X_{i}\right\rangle\left\langle A^{v}\left(X_{j}\right), X_{j}\right\rangle-\left(\left\langle A^{v}\left(X_{i}\right), X_{j}\right\rangle\right)^{2}\right)=n^{2}(\langle\vec{H}, v\rangle)^{2}-\left\|A^{v}\right\|^{2} .
$$

С другой стороны, очевидно, что

$$
\sum_{i=1}^{n}\left(\left\langle\nabla_{X_{i}} v, \xi\right\rangle\right)^{2}=\|\nabla v\|^{2}
$$

Поэтому окончательно имеем

$$
\begin{aligned}
A^{\prime \prime}(0)= & \int_{\mathcal{M}} \\
\quad & \bar{\nabla}^{2} \alpha(v, v)-2 n\langle\bar{\nabla} \alpha, v\rangle H\langle v, \xi\rangle \\
& +\alpha\left(n^{2}\langle H, v\rangle^{2}-\left\|A^{v}\right\|^{2}+\|\nabla v\|^{2}\right) d \mathcal{M} .
\end{aligned}
$$

Здесь через $\bar{\nabla}^{2} \alpha$ обозначена форма второго дифференциала функции $\alpha$.

Перейдем ко второй части доказательства. Прежде всего напомним формулу [19, т. 1 , приложение 6] для дивергенции

$$
L_{V} \omega=(\operatorname{div} V) \omega
$$

справедливую для произвольного гладкого векторного поля $V$ и формы объема $\omega$ произвольного гладкого риманова многообразия. Пусть $e_{1}, \ldots, e_{n+1}-$ 
стандартный ортонормированный базис в $\mathbb{R}^{n+1}$. Положим $e_{k}^{*}=d F_{t}\left(e_{k}\right)$, $k=1, \ldots, n+1$. По построению ясно, что

$$
L_{V} e_{k}^{*}=0 \text {. }
$$

Пусть $\Omega_{1}(t)=F_{t}\left(\Omega_{1}\right)$. Тогда в силу формулы замены переменной имеем

$$
G(t)=\int_{\Omega_{1}(t)} \varphi \omega\left(e_{1}, e_{2}, \ldots, e_{n+1}\right)=\int_{\Omega_{1}} \varphi \omega\left(e_{1}^{*}, e_{2}^{*}, \ldots, e_{n+1}^{*}\right) .
$$

Дифференцируя, будем иметь

$$
G^{\prime}(t)=\int_{\Omega_{1}} \bar{\nabla}_{V} \varphi \omega\left(e_{1}^{*}, e_{2}^{*}, \ldots, e_{n+1}^{*}\right)+\int_{\Omega_{1}} \varphi L_{V} \omega\left(e_{1}^{*}, e_{2}^{*}, \ldots, e_{n+1}^{*}\right) .
$$

Согласно соотношениям (11) и (10) получим

$$
\begin{aligned}
G^{\prime}(t) & =\int_{\Omega_{1}} \bar{\nabla}_{V} \varphi \omega\left(e_{1}^{*}, e_{2}^{*}, \ldots, e_{n+1}^{*}\right)+\int_{\Omega_{1}} \operatorname{div} V \omega\left(e_{1}^{*}, e_{2}^{*}, \ldots, e_{n+1}^{*}\right) \\
& =\int_{\Omega_{1}} \operatorname{div}(\varphi V) \omega\left(e_{1}^{*}, e_{2}^{*}, \ldots, e_{n+1}^{*}\right) .
\end{aligned}
$$

Аналогично,

$$
G^{\prime \prime}(t)=\int_{\Omega_{1}} \operatorname{div}(\operatorname{div}(\varphi V)) \omega\left(e_{1}^{*}, e_{2}^{*}, \ldots, e_{n+1}^{*}\right) .
$$

Используя формулу Остроградского-Гаусса и условие $\left.V\right|_{\partial \Omega_{1} \backslash \mathcal{M}}=0$, будем иметь

$$
\begin{gathered}
G^{\prime}(0)=\int_{\mathcal{M}} \varphi\langle v, \xi\rangle \\
G^{\prime \prime}(0)=\int_{\mathcal{M}}\langle\bar{\nabla} \varphi, \xi\rangle\langle v, \xi\rangle^{2}+\int_{\mathcal{M}} \varphi \operatorname{div}(\langle v, \xi\rangle \xi)\langle v, \xi\rangle \\
=\int_{\mathcal{M}}\langle\bar{\nabla} \varphi, \xi\rangle\langle v, \xi\rangle^{2}+\int_{\mathcal{M}} \varphi \operatorname{div} \xi\langle v, \xi\rangle^{2}+\int_{\mathcal{M}} \varphi \bar{\nabla}_{\xi}\langle v, \xi\rangle .
\end{gathered}
$$

Последнее слагаемое равно нулю, поскольку по построению $\bar{\nabla}_{V} V=0$. Таким образом,

$$
G^{\prime \prime}(0)=\int_{\mathcal{M}}(\langle\bar{\nabla} \varphi, \xi\rangle-\varphi n H)\langle v, \xi\rangle^{2}
$$

Теорема доказана полностью.

\section{§ 3. Доказательство леммы 1}

ДокаЗАТЕЛЬСтво ЛЕммы 1 . Пусть $h: \mathcal{M} \rightarrow \mathbb{R}-C^{1}$-функция, удовлетворяющая (3). Обозначим через $\nu$ вектор внешней нормали к краю $\partial \mathcal{M}$ в $\mathcal{M}$. Тогда, используя неравенство (6), имеем

$$
\begin{aligned}
0 & =\int_{\partial \mathcal{M}} h^{2} \frac{\langle\nabla u, \nu\rangle}{u} d(\partial \mathcal{M})=\int_{\mathcal{M}} \operatorname{div}\left(h^{2} \frac{\nabla u}{u}\right) d \mathcal{M} \\
& =\int_{\mathcal{M}}\left(h^{2} \frac{\Delta u}{u}\right) d \mathcal{M}-\int_{\mathcal{M}}\left(h^{2} \frac{|\nabla u|^{2}}{u^{2}}\right) d \mathcal{M}+2 \int_{\mathcal{M}} h \frac{\langle\nabla u, \nabla h\rangle}{u} d \mathcal{M}
\end{aligned}
$$




$$
\begin{aligned}
\leqslant & -\int_{\mathcal{M}} h^{2}\|A\|^{2} d \mathcal{M}-\int_{\mathcal{M}}\left(h^{2} \frac{|\nabla u|^{2}}{u^{2}}\right) d \mathcal{M}+2 \int_{\mathcal{M}} h \frac{\langle\nabla u, \nabla h\rangle}{u} d \mathcal{M} \\
= & \int_{\mathcal{M}}|\nabla h|^{2} d \mathcal{M}-\int_{\mathcal{M}} h^{2}\|A\|^{2} d \mathcal{M}-\int_{\mathcal{M}}\left(h^{2} \frac{|\nabla u|^{2}}{u^{2}}\right) d \mathcal{M}+2 \int_{\mathcal{M}} h \frac{\langle\nabla u, \nabla h\rangle}{u} d \mathcal{M} \\
& -\int_{\mathcal{M}}|\nabla h|^{2} d \mathcal{M} \leqslant \int_{\mathcal{M}}|\nabla h|^{2} d \mathcal{M}-\int_{\mathcal{M}} h^{2}\|A\|^{2} d \mathcal{M},
\end{aligned}
$$

что и требовалось доказать.

ДокАЗАТЕЛЬСтво СЛЕДСтвия 1 весьма несложно. Достаточно применить теорему 1 к функции $u(m)=\langle\xi, e\rangle$. Здесь вектор $e$ выбран так, чтобы $u(m)>0$. Для поверхностей, заданных графиком функции, этого всегда можно добиться. Кроме того, известно [22], что функция $u(m)$ в метрике поверхности $\mathcal{M}$ удовлетворяет необходимому уравнению Якоби

$$
\Delta u(m)=-\|A\|^{2} u(m)
$$

\section{§ 4. Доказательство теоремы 2}

Предположим, что поверхность $\mathcal{M}$ является устойчивой. Тогда из условия теоремы и соотношения (4) получаем неравенство

$$
\int_{\mathcal{M}}\left(|\nabla h|^{2}-\|A\|^{2}\right) h^{2} d \mathcal{M} \geqslant 0
$$

выполненное для любой $C^{1}$-гладкой функции $h: \mathcal{M} \rightarrow \mathbb{R}$, которая удовлетворяет условиям (3). Из свойств симметрических матриц несложно вывести неравенство $\|A\|^{2} \geqslant n H^{2}$, поэтому $\|A\|^{2} \geqslant n \inf _{\mathcal{M}} H^{2}$. Неравенство (13) перепишется в виде

$$
\int_{\mathcal{M}}\left(|\nabla h|^{2}-n \inf _{\mathcal{M}} H^{2} h^{2}\right) d \mathcal{M} \geqslant 0 .
$$

Ясно, что это неравенство противоречит условию $\mu(\mathcal{M})<n \inf _{\mathcal{M}} H^{2}$. Теорема 2 доказана.

\section{§5. Доказательство теоремы 3}

Пусть $x^{0}-$ центр масс поверхности $\mathcal{M}$ :

$$
x^{0}=\frac{1}{|\mathcal{M}|} \int_{\mathcal{M}} x d \mathcal{M} .
$$

Тогда очевидны равенства

$$
\int_{\mathcal{M}}\left(x_{i}-x_{i}^{0}\right) d \mathcal{M}=0, \quad i=1,2, \ldots, n+1 .
$$

Поскольку поверхность $\mathcal{M}$ устойчива и $\langle\bar{\nabla} \varphi, \xi\rangle \leqslant 0$, то

$$
\int_{\mathcal{M}}\left(\left|\nabla\left(x_{i}-x_{i}^{0}\right)\right|^{2}-\|A\|^{2}\left(x_{i}-x_{i}^{0}\right)^{2}\right) d \mathcal{M} \geqslant 0 .
$$


Суммируя эти неравенства по $i=1,2, \ldots, n+1$, получим

$$
\sum_{i=1}^{n+1} \int_{\mathcal{M}}\left|\nabla\left(x_{i}-x_{i}^{0}\right)\right|^{2} d \mathcal{M}-\sum_{i=1}^{n+1} \int_{\mathcal{M}}\|A\|^{2}\left(x_{i}-x_{i}^{0}\right)^{2} d \mathcal{M} \geqslant 0 .
$$

Несложно проверяется, что

$$
\sum_{i=1}^{n+1}\left|\nabla x_{i}\right|^{2}=\sum_{i=1}^{n+1}\left|e_{i}\right|^{2}=n .
$$

Теперь, используя неравенство Коши-Буняковского, получаем неравенство

$$
0 \leqslant n|\mathcal{M}|-n\left(\int_{\mathcal{M}} H\left\langle x-x^{0}, \xi\right\rangle d \mathcal{M}\right)^{2} \frac{1}{|\mathcal{M}|} .
$$

Здесь мы также воспользовались очевидным соотношением $\|A\|^{2} \geqslant n H^{2}$ и ввели обозначение $|\mathcal{M}|=\int_{\mathcal{M}} d \mathcal{M}$. Поскольку в метрике поверхности имеет место равенство $\Delta\left(x-x^{0}\right)^{2}=2 n+2 n H\left\langle\xi, x-x^{0}\right\rangle$, то из формулы ОстроградскогоГаусса, используя, что $\partial \mathcal{M}=\varnothing$, будем иметь

$$
2 n|\mathcal{M}|+2 n \int_{\mathcal{M}} H\left\langle x-x^{0}, \xi\right\rangle d \mathcal{M}=0 .
$$

Используя это равенство, получаем

$$
n|\mathcal{M}| \geqslant \frac{n}{|\mathcal{M}|}\left(\int_{\mathcal{M}} H\left\langle\xi, x-x^{0}\right\rangle d \mathcal{M}\right)^{2}=\frac{n}{|\mathcal{M}|}|\mathcal{M}|^{2}=n|\mathcal{M}| .
$$

Следовательно, во всех неравенствах, приведенных выше, должны быть равенства. В частности, $\left\langle x-x^{0}, \xi\right\rangle^{2}=\left|x-x^{0}\right|^{2}$, откуда следует, что $\mathcal{M}$ - сфера. Теорема 3 доказана.

\section{§6. Доказательство теоремы 4}

Для доказательства теоремы нам достаточно указать функцию $h: \mathcal{M} \rightarrow \mathbb{R}$ такую, что $\left.h\right|_{\partial \mathcal{M}}=0, \int_{\mathcal{M}} h d \mathcal{M}=0$, и для которой величина

$$
Q(h)=\int_{\mathcal{M}}|\nabla h|^{2}-\|A\|^{2} h^{2} d \mathcal{M}
$$

будет отрицательной. $\mathrm{C}$ этой целью проведем дополнительные оценки этой величины. Введем обозначения

$$
E(t)=\{x \in \mathcal{M}: f(x)=t\}=\mathcal{M} \cap\left\{x: x_{n+1}=t\right\}
$$

и $f(x)=x_{n+1}$. Требуемую функцию $h$ будем искать в виде $h=h(f(x))$. Согласно формуле Кронрода-Федерера [21, теорема 3.2 .12$]$ и неравенству $\|A\|^{2} \geqslant n H^{2}$ имеем

$$
\begin{aligned}
Q(h) & =\int_{\mathcal{M}}|\nabla h|^{2}-\|A\|^{2} h^{2} d \mathcal{M} \\
& \leqslant \int_{a}^{b}\left(h^{\prime}\right)^{2}(\tau) \int_{E(\tau)}|\nabla f| d E(\tau) d \tau-n H^{2} \int_{a}^{b} h^{2}(\tau) \int_{E(\tau)} \frac{1}{|\nabla f|} d E(\tau) d \tau \\
& =\int_{a}^{b}\left(h^{\prime}\right)^{2}(\tau) J(\tau) d \tau-n H^{2} \int_{a}^{b} h^{2} \tau v(\tau) d \tau
\end{aligned}
$$


Здесь мы ввели обозначения

$$
\begin{aligned}
& J(\tau)=\int_{E(\tau)}|\nabla f| d E(\tau), \\
& v(\tau)=\int_{E(\tau)} \frac{1}{|\nabla f|} d E(\tau)
\end{aligned}
$$

и $d E(t)$ - элемент площади сечения $E(t)$.

Положим

$$
h(t)= \begin{cases}\sin \left(\frac{2 \pi \sigma}{\sigma_{0}}\right), & \text { если } 0 \leqslant \sigma<\frac{\sigma_{0}}{2}, \\ \lambda \sin \left(\frac{2 \pi \sigma}{\sigma_{0}}\right), & \text { если } \frac{\sigma_{0}}{2} \leqslant \sigma \leqslant \sigma_{0},\end{cases}
$$

где

$$
\sigma(t)=\int_{a}^{t} \frac{d \tau}{J(\tau)}, \quad \sigma_{0}=\int_{a}^{b} \frac{d \tau}{J(\tau)},
$$

а положительная постоянная $\lambda$ выбрана таким образом, что

$$
\int_{\mathcal{M}} h d \mathcal{M}=\int_{a}^{b} h(\tau) v(\tau) d \tau=0
$$

Существование такой постоянной очевидно по построению функции $h(t)$. Тогда после замены переменной в однократном интеграле будем иметь

$$
\begin{aligned}
Q(h) \leqslant( & \left.\frac{2 \pi}{\sigma_{0}}\right)^{2} \int_{0}^{\sigma_{0} / 2} \cos ^{2}\left(\frac{2 \pi \sigma}{\sigma_{0}}\right) d \sigma-n H^{2} \int_{0}^{\sigma_{0} / 2} \sin ^{2}\left(\frac{2 \pi \sigma}{\sigma_{0}}\right) v(\tau(\sigma)) J(\tau(\sigma)) d \sigma \\
& +\lambda^{2}\left(\frac{2 \pi}{\sigma_{0}}\right)^{2} \int_{\sigma_{0} / 2}^{\sigma_{0}} \cos ^{2}\left(\frac{2 \pi \sigma}{\sigma_{0}}\right) d \sigma \\
& -\lambda^{2} n H^{2} \int_{\sigma_{0} / 2}^{\sigma_{0}} \sin ^{2}\left(\frac{2 \pi \sigma}{\sigma_{0}}\right) v(\tau(\sigma)) J(\tau(\sigma)) d \sigma
\end{aligned}
$$

В силу неравенства Коши-Буняковского $(n-1)$-мерная площадь $L(t)$ сечения $E(t)$ может быть оценена следующим образом:

$$
L(t)=\int_{E(t)} d E(t) \leqslant\left(\int_{E(t)} \frac{1}{|\nabla f|} d E(t)\right)^{1 / 2}\left(\int_{E(t)}|\nabla f| d E(t)\right)^{1 / 2}=\sqrt{v(t) J(t)} .
$$

Поэтому, полагая $L_{\max }=\max _{[a, b]} L(t), L_{\min }=\min _{[a, b]} L(t)$, будем иметь

$$
\begin{aligned}
Q(h) \leqslant & \left(\frac{2 \pi}{\sigma_{0}}\right)^{2} \int_{0}^{\sigma_{0} / 2} \cos ^{2}\left(\frac{2 \pi \sigma}{\sigma_{0}}\right) d \sigma-L_{\min }^{2} n H^{2} \int_{0}^{\sigma_{0} / 2} \sin ^{2}\left(\frac{2 \pi \sigma}{\sigma_{0}}\right) d \sigma \\
& +\lambda^{2}\left(\frac{2 \pi}{\sigma_{0}}\right)^{2} \int_{\sigma_{0} / 2}^{\sigma_{0}} \cos ^{2}\left(\frac{2 \pi \sigma}{\sigma_{0}}\right) d \sigma-L_{\min }^{2} \lambda^{2} n H^{2} \int_{\sigma_{0} / 2}^{\sigma_{0}} \sin ^{2}\left(\frac{2 \pi \sigma}{\sigma_{0}}\right) d \sigma \\
= & \frac{\sigma_{0}}{4}\left(1+\lambda^{2}\right)\left(\left(\frac{2 \pi}{\sigma_{0}}\right)^{2}-n H^{2} L_{\min }^{2}\right) .
\end{aligned}
$$


Заметим, что

$$
\sigma_{0}=\int_{a}^{b} \frac{d t}{J(t)} \geqslant \int_{a}^{b} \frac{d t}{L(t)} \geqslant \frac{b-a}{L_{\max }}
$$

Таким образом,

$$
Q(h) \leqslant \frac{\sigma_{0}}{4}\left(1+\lambda^{2}\right) L_{\max }^{2}\left(\left(\frac{2 \pi}{b-a}\right)^{2}-\frac{L_{\min }^{2}}{L_{\max }^{2}} n H^{2}\right)<0
$$

в силу условия теоремы. Вторая часть теоремы доказывается точно так же, только к равенству (14) необходимо применить оценку $\|A\|^{2} \geqslant n^{2} H^{2}$, справедливую для поверхностей неотрицательной скалярной кривизны. Теорема доказана.

ЗАмЕЧАниЕ 2. Как было показано и экспериментально проверено в [20, $\S 1.4]$, жидкий цилиндр неустойчив тогда и только тогда, когда его высота строго больше длины окружности основания. Последнее следует из неравенства (7).

\section{Список литературы}

1. Barbosa J. L., do Carmo M., "On the size of a stable minimal surface in $\mathbb{R}^{3 ",}$ Amer. J. Math., 98:2 (1976), 515-528.

2. Barbosa J.L., do Carmo M., "Stability of minimal surfaces and eigevalues of the Laplacian", Math. Z., 173:1 (1980), 13-28.

3. do Carmo M., Peng C. K., "The stable minimal surfaces in $\mathbb{R}^{3}$ are planes", Bull. Amer. Math. Soc. (N. S.), 1:6 (1979), 903-906.

4. Lawson H.B., "Some intrinsic characterizations of minimal surfaces", J. Analyse Math., 24 (1971), 151-161.

5. Фоменко А. Т., "О скорости роста и наименьших объемах глобально минимальных поверхностей в кобордизмах", Тр. семинара по вект. и тенз. анализу, Bып. 21, МГУ, М., 1985, 3-12.

6. Тужилин А.А., Фоменко А. Т., Элементы геометрии и топологии минималъных поверхностей, Наука, М., 1991.

7. Тужилин А.А., "Индексы типа Морса двумерных минимальных поверхностей в $\mathbb{R}^{3}$ и $H^{3 ”,}$ Изв. АН СССР. Сер. матем., 55:3 (1991), 581-607.

8. Погорелов А. В., "Об устойчивости минимальных поверхностей”, Докл. АН СССР, 260:2 (1981), 293-295.

9. Hoffman D., Osserman R., "The area of generalized Gaussian image and the stability of minimal surfaces in $S^{n}$ and $\mathbb{R}^{n "}$, Math. Ann., 260:4 (1982), 437-452.

10. Клячин В.А., Миклюков В.М., "Об одном емкостном признаке неустойчивости минимальных гиперповерхностей”, Докл. РАН, 330:4 (1993), 424-426.

11. Finn R., Vogel T. I., "On the volume infimum for liquid bridges", Z. Anal. Anwend., 11:1 (1992), 3-23.

12. Langbein D., "Stability of liquid bridges between parallel plates", Microgravity Sci. Techn., 5 (1992), 2-11.

13. Concus P., Finn R., McCuan J., "Liquid bridges, edges blobs, and Scherk-type capillary surfaces", Indiana Univ. Math. J., 50:1 (2001), 411-440.

14. Vogel T.I., "Types of instability for the trapped drop problem with equal contact angles", Geometric Analysis and Computer Graphics, Math. Sci. Res. Inst. Publ., 17, eds. P. Concus, R. Finn, D. Hoffman, Springer-Verlag, New York, 1991, 195-203.

15. Финн Р., Равновесные капиллярные поверхности. Математическал теория, Мир, M., 1989. 
16. Wente H. C., "The stability of the axially symmetric pendant drop", Pacific J. Math., 88:2 (1980), 421-470.

17. Клячин В.А., "Об устойчивости и неустойчивости поверхностей предписанной средней кривизны", Докл. РАН, 403:6 (2005), 739-741.

18. Александров А.Д., "Теоремы единственности для поверхностей в целом. V", Вестн. ЛГУ, 13:19 (1958), 5-8.

19. Кобаяси Ш., Номидзу К., Основы дифференииалъной геометрии, в 2-х т. T. 1, 2, Наука, М., 1981.

20. Саранин В. А., Равновесие жидкостей и его устойчивость, Ин-т компьютерных исследований, М., 2002.

21. Федерер Р., Геометрическая теория меры, Наука, М., 1984.

22. Simons J., "Minimal varieties in riemannian manifolds", Ann. of Math.(2), 88 (1968), 62-105.

В. А. Клячин (V.A. КLYAChin)

Волгоградский государственный университет

E-mail: klchnv@mail.ru
Поступило в редакцию 23.09.2005 